\title{
Journal of Community Medicine \& Health Education
}

\section{Study of the Acceptance and Perceived Efficacy of Animal Assisted Therapy (AAT) for Parents and Nurses in the Psychiatry Unit of Meyer Children's Hospital in Florence - Italy}

\author{
Stefanini MC ${ }^{1 *}$, Bigalli $\mathrm{E}^{1}$ and Tani $\mathrm{F}^{2}$ \\ ${ }^{1}$ Neurofarba Department, Meyer Children's Hospital, University of Florence, Italy \\ ${ }^{2}$ Department of Health Sciences, Psychology and Psychiatry Unit, University of Florence, Italy
}

"Corresponding author: Prof. Maria Cristina Stefanini, Neurofarba Department, Meyer Children's Hospital, University of Florence, Viale Pieraccini, Firenze, 6- 50129, Italy, Tel: +39-055-4296194; Fax: +39-055-4296190; E-mail: mariacristina.stefanini@unifi.it

Received date: April 25, 2016; Accepted date: June 23, 2016; Published date: June 30, 2016

Copyright: $\odot 2016$ Stefanini MC, et al. This is an open-access article distributed under the terms of the Creative Commons Attribution License, which permits unrestricted use, distribution, and reproduction in any medium, provided the original author and source are credited.

\begin{abstract}
Objectives: This short paper is part of wider research aimed at comparing the effects of Animal-Assisted Therapy (AAT) with a standard treatment protocol in 20 children and adolescents admitted to psychiatry hospitals for severe mental breakdown (with RCT). After analysing the global results we concluded that the benefits could not only be described in terms of direct effectiveness and care outcomes, but there were also other factors that we should consider, in particular in relation to the context of care. The focus was to analyze those factors, because the results of this treatment were quite distinctive and specific to AAT. The main purpose of this study was to examine the extent to which parents and nurses accept the effects of AAT and perceive its efficacy.
\end{abstract}

Method: For this purpose, at the end of the AAT treatment, we gave a specially formulated questionnaire to both parents and nurses.

Results: Data analyses showed that nurses evaluate AAT to be very efficient in improving both the adolescent's quality of stay in hospital and also their compliance with the care provided. Parents expressed a strong acceptance of AAT and evaluated this treatment as an efficient way to both improve the way in which their children express emotions and also facilitate their social skills.

Conclusion: The study confirms, in a wider context, the results of our previous studies, not only on the clinical efficacy of AAT, but also on the perceived efficacy for parents and hospital staff and in the general context of care. This underlines the importance to further analyze the factors related to the environment and document the indirect benefits related to a more positive care environment.

Keywords: Animal - assisted therapy; Hospitalized adolescent psychiatric patients; Acceptance; Perceived efficacy questionnaire for parents and nurses

\section{Introduction}

AAT is considered a therapy that integrates, enhances and supports the treatments normally provided for many diseases. In particular, AAT aims to integrate treatments that deal with improving physical, social, emotional and / or cognitive functions [1,2]. AAT involves the interaction between the patient and a trained animal that has been specially educated in this therapy, with the support of a pet-partner and a mental health operator with the aim of facilitating progress towards therapeutic goals [3-7].

In the hospital setting, there are several studies that have examined the benefits of AAT in children, particularly in the pediatric population [8-10]. The reported results suggest that, after individual sessions of AAT, the patient displayed a short-term capacity to reduce the stress response and an improvement in mood $[11,12]$.

With regards to psychological and psychiatric problems, up to now, studies have been mainly carried out on children with autism $[13,14]$ mental retardation and chronic mental illness-related diseases [15,16] or a long-standing illness, such as cancer $[17,18]$. At the moment, several studies are indicating that the benefits for clinical problems are also possible during psychiatric hospitalization for children or adolescents [19].

\section{Context of the Study and General Methodology of Research}

This study was carried out in collaboration with the Guide Dogs for the Blind Schools in the Tuscany Region, (pet-partners are trained for Animal-Assisted Activities and AAT interventions to the standards of Pet-Partner ${ }^{\oplus}$, ex-Delta Society).

The research was carried out within the Child and Adolescent Psychiatric Unit, in Meyer Children's Hospital in Florence. This Unit treats young patients with a broad range of acute psychiatric disorders (mood disorders, anxiety disorders and eating disorders). Patients were hospitalized for an extensive period of time, generally from 2 weeks to 3-4 months (either as out-patients in the Day Hospital or through inpatient hospitalization). During hospitalization, the therapeutic program includes interventions for the patient, his/her family and also in relation to the context of his/her lifestyle. The main therapeutic 
Citation: Stefanini MC, Bigalli E, Tani F (2016) Study of the Acceptance and Perceived Efficacy of Animal Assisted Therapy (AAT) for Parents and Nurses in the Psychiatry Unit of Meyer Children's Hospital in Florence - Italy. J Community Med Health Educ 6: 448. doi: $10.4172 / 2161-0711.1000448$

Page 2 of 4

purposes, in the hospital environment, are aimed at limiting the damage of acute psychic failure and safeguarding the patients from excessive external stimuli while at the same time continuing to offer a minimum experience of everyday life (school, external social activities, supportive relationships, etc.). Since 2011, Animal-Assisted Activities (AAA) and AAT have been carried out within this Unit and the effectiveness of this kind of treatment has been studied in depth [20].

We used a methodology with high quality standards for AAT research. We used a pre-post experimental design (T0-T1) with a randomized controlled trial (RCT); 20 patients who underwent AAT were compared with 20 patients who received standard therapeutic treatment. In our analyses, we focused on: the improvement in clinical status using global functioning, measured with the Children Global Assessment Scale (C GAS) [The Children Global Assessment Scale-C GAS [21] provides a measurement of global functioning in children and adolescents (6-17 years old), with a rating scale ranging from 0 to 100 points], the format of care and the regular school attendance measured by a rating scale; we found that there were significant differences between the treatment and control groups with changes occurring from $\mathrm{T} 0$ to $\mathrm{T} 1$, in particular, there was a major increase in the format of hospital care $(\mathrm{t}(32)=2.41, \mathrm{p}=0.02)$, regular school attendance $(\mathrm{t}(32)=2.25, \mathrm{p}<0.03)$, and global functioning $(\mathrm{t}(32)=4.57$, $\mathrm{p}<0.0001)$, in the treatment group [20] using the Italian version of Youth Self Report (YSR) [by [22] a self-report questionnaire designed for children and adolescents from 11 to 18 years old, comprising 119 clinical items], and these results indicated a significant decrease in internalizing symptoms $(\mathrm{p}<0.001)$ and an increase in total competence $(\mathrm{p}<0.001)$, as well significant improvements in global functioning $(\mathrm{p}<0.0001)$ in the AAT treatment group, but not in the control group $[20,23]$.

\section{Objects of this Study}

After analysing the global results we concluded that the benefits could not only be described in terms of direct effectiveness and care outcomes, but there were also other factors that needed to be taken into consideration, in particular in relation to the context of care. It improves the working environment for the ward staff and the relationships with family members who spend a lot of time on the ward with their children. In an effort to document this data we drew up and distributed specially formulated questionnaires. Through these questionnaires, the present study examined the satisfaction, the acceptance and the perceived effectiveness of AAT in parents and nurses after 3 months of AAT treatment.

\section{Participants and Methodology}

For this purpose, the specially formulated questionnaire was given to the parents of 20 psychiatric adolescent patients who had undergone a session of AAT during their period of hospitalization. The patients were 9 males and 11 females, aged from 11 to 17 years old, with severe psychiatric diagnoses. The questionnaire consisted of 10 questions aimed to assess how, according to the parents, the treatment of AAT was received by their children and the perceptions and evaluations on the efficacy of this treatment.

At the same time, a different questionnaire was given to the ward nurses. This questionnaire consisted of 6 questions about the perceived changes they had seen in the patients who had undergone AAT and the problems they had encountered. Both parents and nurses' questionnaires are reported in Tables 1 and 2.
The analysis of the data was carried out by calculating the percentage frequencies of each item.

1. What impact has the AAT experience had on the ward?

Better emotional climate in the ward

Greater personal involvement in taking charge of the patient

Improved patient/nurse time ratio.

Enhancing knowledge and understanding of the patient

Difficulties in interacting with the different people involved

Difficulty in understanding the objectives of AAT

2. Do you think that AAT for patients has contributed to:

Increased communication between patients

Increased communication between patients and staff

The encouragement to create relations

Improved self-esteem

Increased emotional expression

3. What were the reactions of the parents?

curiosity - participation - indifference - opposition

4. Have you experienced difficulties during AAT?

To manage the information

To operate shifts

To integrate the daily routine into ward life

The limited involvement of the patients

5. How would you rate the overall organization of AAT?

poor - insufficient - sufficient - good - very good - excellent

6. Do you think that continued AAT in the ward would be useful? Y/N

Table 1: AAT satisfaction questionnaire-nurses.

1. Had you ever heard of AAT? Y/N

2 What do you think about children having contact with animals in the hospital?

In favour-slightly in favour-not in favour-don't know

3. Do you believe that AAT can be beneficial for young patients? $\mathrm{Y} / \mathrm{N}$

4. If Yes: What kind of benefits does it bring?

5. Do you think that AAT has brought about a change in your child? Y/N

6 What does AAT mean for your child?

A way to accept admission to hospital more willingly

A way to socialize with other kids in the ward

A way to facilitate emotional expression

An efficient way to get better

7. In your opinion, how does your child perceive this type of activity? 
Citation: Stefanini MC, Bigalli E, Tani F (2016) Study of the Acceptance and Perceived Efficacy of Animal Assisted Therapy (AAT) for Parents and Nurses in the Psychiatry Unit of Meyer Children's Hospital in Florence - Italy. J Community Med Health Educ 6: 448. doi: 10.4172/2161-0711.1000448

Page 3 of 4

8. How enthusiastically did your child participate in AAT?

Not at all - a little-somewhat - quite a lot-a lot

9 Do you think that continued AAT in the ward would be useful Y/N

10. Do you have any suggestions as to how to improve the quality of this project?

Table 2: AAT satisfaction questionnaire-parents.

\section{Results}

The results showed general satisfaction with AAT, both from nurses and parents. In particular, nurses perceived the significant way AAT had contributed towards the improvement in the young patients' compliance to care and their greater acceptance of treatment (f.4). Moreover, they perceived AAT as an efficient way to facilitate emotional expression (f.2), and the social relations of patients (f.2) (Figure 1). We selected this item because it seemed relevant to the daily work of nurses. Furthermore, AAT could be considered an added burden for healthcare staff, when compared to other forms of treatment but the data from our study excluded this.

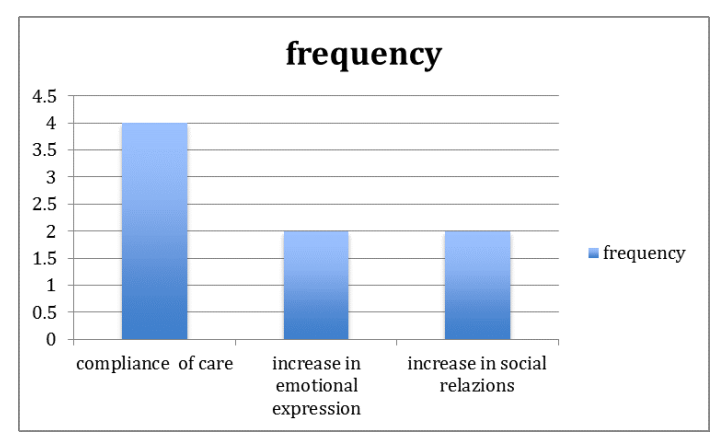

Figure 1: Nurses's perception at AAT's contribution - question 2.

In the same way, the parents of the adolescents who received AAT perceived, in their children, a greater acceptance of the hospital experience, clinical practices and treatments (f.4) and a significant improvement in their emotional expression (f.3) and social skills (f.3) (Figure 2). We selected this item because it seemed important for the therapeutic alliance and collaborative interactions within the ward. This data may also be significant in promoting positive parental mirroring, which is one of the basic objectives of hospital intervention.

\section{Conclusion}

This data confirms and extends the context of the results that were previously obtained in other studies.

Indeed, we observed that the benefits of AAT in hospital could not only be described in terms of direct effectiveness and care outcomes, but there were also other factors that needed to be taken into consideration, in particular in relation to the context of care. We believe that this aspect is distinctive and specific to this type of treatment in very real terms.

Qualitative analyses of this study, aimed at analyzing the acceptance and efficacy perceived by parents and hospital staff, verified that even nurses and parents have recognized the effectiveness and the improvements of some specific parameters of young patients, in particular the compliance to care and the capacity for emotional expression after the intervention of AAT.

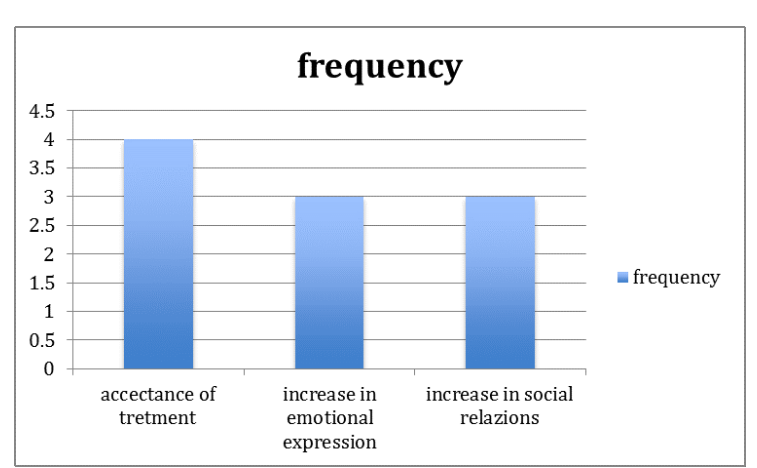

Figure 2: Parent's perception at AAT's contribution - question 6.

We believe that this aspect is particularly important and specific to AAT, and that it is characterized by the broad-resonance effects on the environment, the promotion of social and relational aspects, normalizing, and the experiences of positive emotional sharing. We can identify positive environmental mirroring and enrichment as one of the mechanisms of the effectiveness of AAT.

We need to further analyze the factors related to the environment and document the indirect benefits related to a more positive care environment.

\section{References}

1. Cole KM, Gawlinski A, Steers N, Kotlerman J (2007) Animal-assisted therapy in patients hospitalized with heart failure. Am J Crit Care 16: 587-585.

2. Parish-Plass N (2008) Animal-Assisted Therapy with Children Suffering from Insecure Attachment Due to Abuse and Neglect: A Method to Lower the Risk of Intergenerational Transmission of Abuse? Clin Child Psychol Psychiatry 13: 7-30.

3. Barker SB, Pandurangi AK, Best AM (2011) Effects of animal-assisted therapy on patients' anxiety, fear, and depression before ECT. J ECT 19: 38-44.

4. Hoffman AOM, Lee AH, Wertenauer F, Lang UE (2009) Dog-assisted intervention reduce anxiety in hospitalized patients with major depression. Euro J Integrative Med 1: 145-148.

5. Mallon G (1992) Utilization of animals as therapeutic adjuncts with children and youth: A review of the literature. Child and Youth Care Forum 21: 53-67.

6. Cirulli F, Borgi M, Berry A, Francia N, Alleva E (2011) Animal-assisted interventions as innovative tools for mental health. Ann Ist Super Sanità 47: 341-348.

7. Kamioka H, Okada S, Tsutani K, Park H, Okuizumi H, et al. (2014) Effectiveness of animal-assisted therapy: A systematic review of randomized controlled trials. Complement Ther Med 22: 371-390.

8. Balluerka N, Muela A, Amiano N, Caldentey MA (2014) Influence of animal-assisted therapy (AAT) on the attachment representations of youth in residential care. Children and Youth Services Review 42: 103-109.

9. Endenburg N, van Lith HA (2011) The influence of animals on the development of children. Vet J 190: 208-214.

10. Canino G, Costello JE, Angold A (1999) Assessing functional impairment and social adaptation for child mental health service research: A review of measures. Mental Health Services Research 1: 93-108. 
Citation: Stefanini MC, Bigalli E, Tani F (2016) Study of the Acceptance and Perceived Efficacy of Animal Assisted Therapy (AAT) for Parents and Nurses in the Psychiatry Unit of Meyer Children's Hospital in Florence - Italy. J Community Med Health Educ 6: 448. doi: $10.4172 / 2161-0711.1000448$

Page 4 of 4

11. Kaminski M, Pellino T, Wish J (2002) Play and Pets: The Physical and Emotional Impact of Child-Life and Pet Therapy on Hospitalized Children. Children's Health Care 31: 321-335.

12. Melson GF, Fine AH (2010) Animal in the lives of children. Hand- book on animal-assisted therapy. Academic Press, New York, pp: 223-246.

13. Ming Lee, Yeh A (2008) Canine AAT model for autistic children. At Taiwan International Association of Human-Animal Interaction International Conference, Japan.

14. Martin F, Farnum J (2002) Animal-assisted therapy for children with pervasive developmental disorders. West J Nurs Res 24: 657-670.

15. Reed R, Ferrer L, Villegas N (2012) Natural healers: A review of animal assisted therapy and activities as complementary treatment for chronic conditions. Rev Lat Am Enfermagem 20: 612-618.

16. Wu AS, Niedra R, Pendergast L, McCrindle BW (2002) Acceptability and impact of pet visitation on a pediatric cardiology inpatient unit. J Pediatr Nurs 17: 354-362.

17. Urbanski BL, Lazenby M (2012) Distress among hospitalized pediatric cancer patients modified by pet-therapy intervention to improve quality of life. J Pediatr Oncol Nurs 29: 272-282.
18. Orlandi M, Trangeled K, Mambrini A, Tagliani M, Ferrarini A, et al. (2007) Pet therapy effects on oncological day hospital patients undergoing chemotherapy treatment. Anticancer Res 27: 4301-4303.

19. Terzian M, Hamilton K, Ericson S (2011) What works to prevent or reduce internalizing problems or socio-emotional difficulties in adolescents. Child trends.

20. Stefanini MC, Martino A, Allori P, Galeotti F, Tani F (2015) The use of Animal-Assisted Therapy in adolescents with acute mental disorders: A randomized controlled study. Complementary Therapy in Clin Pract 21: 42-46.

21. Schaffer D, Gould MS, Brasic J, Ambrosini P, Fisher P, et al. (1983) A children's global assessment scale (CGAS). Arch Gen Psychiatry 40: 1228-1231.

22. Achenbach T (1991) Manual for the Youth Self-Report and 1991 Profile. Project on Human development in Chicago Neighborhoods.

23. Stefanini MC, Martino A, Bacci B, Tani F (2016) The Effect of AnimalAssisted Therapy on Emotional and Behavioral Symptoms in Children and Adolescents Hospitalized for Acute Mental Disorders. EUJIM Online publication. 